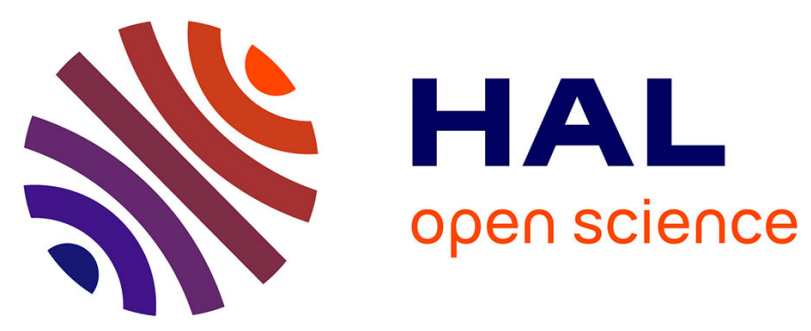

\title{
Time-lapse scanning surface plasmon microscopy of living adherent cells with a radially polarized beam
}

Lotfi Berguiga, Laura Streppa, Elise Boyer-Provera, Cristina Martinez-Torres, Laurent Schaeffer, Juan Elezgaray, Alain Arneodo, Françoise Argoul

\section{- To cite this version:}

Lotfi Berguiga, Laura Streppa, Elise Boyer-Provera, Cristina Martinez-Torres, Laurent Schaeffer, et al.. Time-lapse scanning surface plasmon microscopy of living adherent cells with a radially polarized beam. Applied optics, 2016, 55 (6), pp.1216-1227. 10.1364/AO.55.001216.v001 . hal-01279128

\section{HAL Id: hal-01279128 \\ https://hal.science/hal-01279128}

Submitted on 25 Feb 2016

HAL is a multi-disciplinary open access archive for the deposit and dissemination of scientific research documents, whether they are published or not. The documents may come from teaching and research institutions in France or abroad, or from public or private research centers.
L'archive ouverte pluridisciplinaire HAL, est destinée au dépôt et à la diffusion de documents scientifiques de niveau recherche, publiés ou non, émanant des établissements d'enseignement et de recherche français ou étrangers, des laboratoires publics ou privés.

\section{다(1)(2)}

Distributed under a Creative Commons Attribution - ShareAlikel 4.0 International 


\title{
Time-lapse scanning surface plasmon microscopy of living adherent cells with a radially polarized beam
}

\author{
Lotfi Berguiga, ${ }^{2}$ Laura Streppa, ${ }^{1}$ Elise Boyer-Provera, ${ }^{1}$ Cristina Martinez-Torres, ${ }^{1}$ \\ Laurent Schaeffer, ${ }^{3}$ Juan Elezgaray, ${ }^{4}$ Alain Arneodo, ${ }^{1,5}$ and Françoise Argoul ${ }^{1,5, *}$ \\ ${ }^{1}$ CNRS UMR5672, LPENSL, Université de Lyon, ENS de Lyon, 46 Allée d'Italie, 69001 Lyon, France \\ ${ }^{2}$ CNRS USR3010, LJC, Université de Lyon, ENS de Lyon, 46 Allée d'Italie, 69001 Lyon, France \\ ${ }^{3}$ CNRS UMR5239, LBMC, Université de Lyon, ENS de Lyon, 46 Allée d'Italie, 69001 Lyon, France \\ ${ }^{4}$ CNRS UMR 5248, CBMN, Université Bordeaux, Allée Geoffroy Saint Hilaire, Bât B14, 33600 Pessac, France \\ ${ }^{5}$ CNRS UMR 5798, LOMA, Université Bordeaux, 351 Cours de la Libération, 33405 Talence, France \\ *Corresponding author: fargoul@u bordeaux.fr
}

\begin{abstract}
We report on a fibered high-resolution scanning surface plasmon microscope for long term imaging of living adherent cells. The coupling of a high numerical aperture objective lens and a fibered heterodyne interferometer enhances both the sensitivity and the long term stability of this microscope, allowing for time-lapse recording over several days. The diffraction limit is reached with a radially polarized illumination beam. Adherence and motility of living C2C12 myoblast cells are followed for $50 \mathrm{~h}$, revealing that the dynamics of these cells change after $10 \mathrm{~h}$. This plasmon enhanced evanescent wave microscopy is particularly suited for investigating cell adhesion, since it can not only be performed without staining of the sample but it can also capture in real time the exchange of extracellular matrix elements between the substrate and the cells.
\end{abstract}

OCIS codes: (240.6680) Surface plasmons; (180.5810) Scanning microscopy; (170.3880) Medical and biological imaging; (120.3180) Interferometry; (060.2310) Fiber optics; (110.4280) Noise in imaging systems.

\section{INTRODUCTION}

During the past decade, the evidence has become undeniable that in adhesion related processes the behavior of cells is not only controlled by biochemical cues but also involves many physical determinants like their structural and dynamical prop erties driven by molecular motors. The adhesion of living cells has an impact on their migration, division or death, differen tiation, and surrounding tissue development [1]. It is governed by molecular interactions of the cytoskeleton (CSK) with the extracellular matrix $(\mathrm{ECM})$ via signal transduction receptors (integrins and discoidin domain tyrosine kinase receptors) [2,3] and cytokine exchanges (growth factors) [4]. Integrin mediated adhesion is a complex cascade of biochemical and biomechanical events, for instance integrin binding to fibronec tin (Fn) involves a conformational change (activation) of its Fn receptor that results in a mechanical coupling [5]. Bound Fns then rapidly associate with the actin CSK and cluster together, giving rise to adhesion complexes that contain cytoplasmic structural proteins such as vinculin and talin, and signaling molecules like focal adhesion kinase (FAK), Ras, and Src
[6,7]. Thanks to bidirectional mechanochemical mechanisms and their leading edge protrusions (lamellipodia and filopodia), living cells sense the stiffness of their environment and retro actively adjust their spreading, contraction, and migration [8]. Once driven to adhesion, they can choose among different types of adhesion interactions, mediated by proteins [9]. These include focal adhesions, close contacts, and ECM con tacts (fibrillar adhesion). The strongest of these are focal adhe sions, which have a tight contact within a distance of only $1020 \mathrm{~nm}$ to the substrate. Close contacts often surround focal adhesions, but have a larger gap distance of about $3050 \mathrm{~nm}$. ECM contacts are long range adhesions with distances greater than $100 \mathrm{~nm}$ between the cell and the substrate. We choose in this work C2C12 myoblast cells for characterizing their adhe sion and migration on gold surfaces. C2C12 is an immortalized mouse myoblast cell line that readily proliferates in high serum conditions, and differentiates and fuses in low serum condi tions. It is a very useful system to study aspects of myogenesis, metabolism, and muscle biology [10]. C2C12 myoblast cell adhesion and motility are critical for their myogenesis. 
During differentiation, these cells become more fusiform and aligned, exhibiting more intercellular contacts and cell membrane fusions leading to elongated contractile syncytia multinucleated myotubes [10].

High resolution characterization of living cell substrate in teraction was achieved for the first time to our knowledge by total internal reflection microscopic (TIRM) observation of the cell substratum interface $[11,12]$. Combining TIRM and interferometric methods, interference reflection microscopes (IRMs) improved their sensitivity, allowing quantitative estima tion of the cell substrate gap distances and, importantly, released the sample preparation from harmful staining proce dures $\left[\begin{array}{ll}13 & 15\end{array}\right]$. Due to its high sensitivity to refractive index (RI) changes at metal dielectric interfaces, surface plasmon res onance (SPR) was first introduced in the biology community as a biosensor for probing biochemical interactions and kinetics [16]. This optical technique was adapted subsequently for SPR imaging (SPRi) of cellular adhesion. Exploiting the ampli fication of surface plasmon polaritons (SPPs) [17] occurring on a metal dielectric interface, living cell SPRi was shown to pro vide a reliable estimate of cell surface distances at focal adhesion sites and close contacts [18]. SPRi was also used for long term imaging of protein salting out and cell substrate interactions $[19,20]$. Prism coupled SPRi was limited by the lateral propa gation of SPPs, making cell details inaccessible. The introduc tion of high numerical aperture (NA) objective lenses [ [21 23] raised SPRi to the level of IRM and TIRM, up to what is called nowadays high resolution surface plasmon microscopy (HRSPM). Objective coupled SPPs are localized SPPs. En hancement of the sensitivity and resolution of HRSPMs was improved in scanning versions of these microscopes by intro ducing interferometers [24,25] and confocal imaging [26 28]. Wide field (nonscanning) devices were also developed with (i) an annulus to confine the incoming light angles around the SPR angle [29] and (ii) a TIRM for focusing the light beam in the back focal plane of the objective lens at the SPR angle [30]. For both methods, the light reflected back from the sample was imaged on a 2D camera. HRSPM has been further applied to observe microstructures [24,31], micro and nanoparticles [23,32 34], bilipid layers [35,36], self assembled monolayers [30], and biomolecular binding events [27,37,38]. Cellular observation with HRSPM was performed on fixed cells in air [39 42] and in a liquid medium [43 45]. However, only a few experiments were carried out on living cells [ 46 49]. In Ref. [48], the strength of adhesion of single SH EP1 epithelial cells with collagen was measured as a function of the osmotic pressure using a TIRF SPRM configuration. However, full HRSPRM was not exploited and cellular observations were limited in time to a few minutes. Lately, better resolution was obtained with a scanning HRSPRM and a radially polarized light by Toma et al., [49] and the cell substrate gaps were esti mated for human embryonic kidney (HEK293) cells and poly L lysine coated gold surfaces, with bare gold as a control.

In this paper, as suggested by Somekh's group [24,25], we use a scanning IRM HRSPRM to observe living mouse myo blast cells adherent on bare gold, with long term $(50 \mathrm{~h})$ time lapse video recording. We actually improve this technique by introducing a radially polarized illumination and a fibered interferometer, both leading to better resolution and stability. We show that the resolution reaches the theoretical diffraction limit and that several days of recording can be achieved with this stabilized and compact setup. To the best of our knowl edge, this is the first time that a scanning HRSPRM (or a scan ning surface plasmon microscope SSPM) is used for long term cell observation in a liquid medium. In the first part of the manuscript, we review all technical issues that contribute to a correct interpretation of the images produced by this optical device. The experimental setup is described with special atten tion to the fully fibered interferometer. The operating condi tions and the contrast mechanism of this scanning HRSPRM are detailed. The long term microscope stability is discussed and the resolution and penetration depth of the microscope are characterized. In the second part, we report on long term (48 h) imaging of living mouse myoblast cells. In particular, we reveal and quantify changes in the cell dynamics and their adhesion properties.

\section{SSPM IN LIQUID}

\section{A. Experimental Setup}

The SSPM principle is sketched in Fig. 1(a). SPPs are excited and confined with a $1.57 \mathrm{NA}$ objective lens (Zeiss). When the objective lens is focused beyond the gold film $(z>0)$, into the liquid medium, SPPs propagate on the gold/liquid interface symmetrically toward the objective axis and, by leakage radia tion (LR), a portion of them return to the back focal plane of the objective lens. The following two kinds of rays contribute to the back reflected light: the LR rays that have excited SPPs and the other rays that have not excited SPPs. Between these two kinds of rays, there is a phase difference due to defocus and
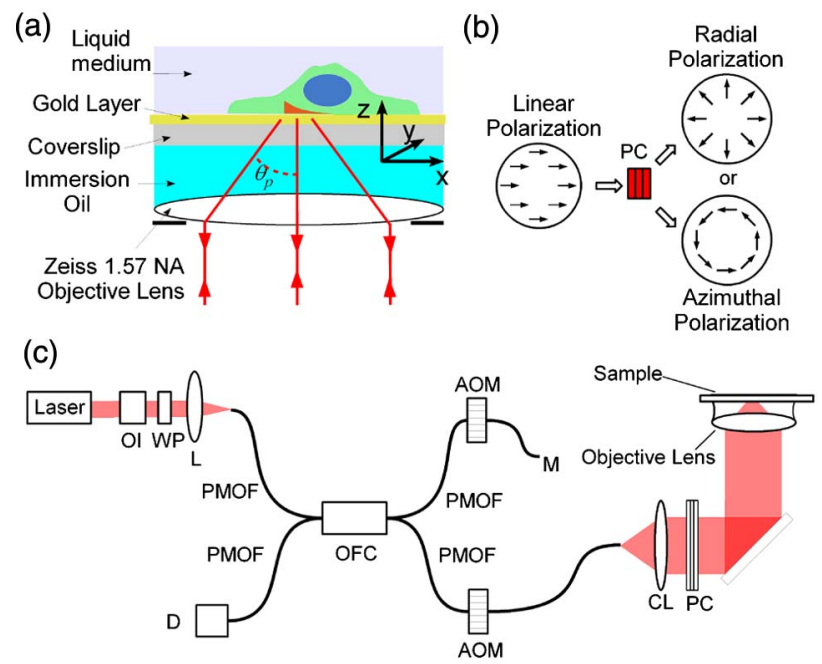

Fig. 1. Scanning surface plasmon microscope setup. (a) Sample con figuration with the SPP excitation through a 1.57 NA (Carl Zeiss Microscopy $\mathrm{GmbH}$ ) objective lens. (b) Spatial distribution of the polarization at the entrance of the objective lens aperture. The polari zation converter (PC) switches between a radially and azimuthally polarized beam. (c) Microscope setup with the fully fibered interfer ometer. OI: optical isolator, WP: half wavelength waveplates, L: lens, PMOF: polarization maintaining optical fiber, OFC: optical fiber cou pler, AOM: acousto optics modulator, D: detector, CL: collimating lens. 
SPP propagation. This phase difference provides information on the SPP excitation conditions in the focused region and is accessed by an interferometric detection method. As SPPs are excited only with $\mathbf{p}$ polarization, the illumination of the objec tive lens aperture with a linearly polarized beam does not allow efficient excitation of SSPs. Actually, for a polar SPR angle $\theta_{p}$ [Fig. 1(a)], only a limited range of azimuthal angle $\varphi$ contrib utes to the SPPs excitation. Given the radial geometry of the objective lens, all azimuthal angles can excite the SPPs $[33,40,43]$ with a radially polarized beam. In this case, the polarization is purely p. For this purpose, we have introduced a liquid crystal cell polarization converter (PC) [50] to convert a linearly polarized beam into a radially polarized beam [Fig. 1(b)]. From this experimental setup, images with or with out SPP excitation can be obtained by switching between ra dially and azimuthally polarized beams with the PC. The optical response of the microscope versus the defocus, known as the $V(z)$ curve, can be expressed in the integral form [24]

$$
V(z) \propto \int_{0}^{2 \pi} \int_{0}^{\theta_{b}} P^{2}(\theta) R(\theta) e^{i 4 \pi n_{1} z \cos \theta / \lambda} \sin \theta \mathrm{d} \theta \mathrm{d} \varphi,
$$

where $P(\theta)$ is the pupil function of the objective lens, $\theta$ and $\varphi$ are the polar and azimuthal angles of the incoming light inside the objective lens, $n_{1}$ the refractive index of the coupling medium (the objective lens, the coverslip and the immersion oil), and $R(\theta)$ is the reflection coefficient of the sample. $R_{p}(\theta)$ [resp. $R_{s}(\theta)$ ] is obtained with a purely $\mathbf{p}$ (resp. $\mathbf{s}$ ) polari zation using a radially (resp. azimuthally) polarized beam.

As illustrated in Fig. 1(c), we have designed a fibered hetero dyne interferometer allowing a better compaction of the experimental setup, together with some shortening of the time consuming optical alignment and adjustment steps. A Gaussian beam of a $1.5 \mathrm{~mW}$ stabilized red He Ne Laser is in jected into the polarization maintaining optical fiber (PMOF). Inside the fiber, the light is separated in amplitude by a $50 / 50$ optical fiber coupler (OFC). In the first arm, the light fre quency is shifted by an acousto optical modulator (AOM) and is reflected at the end of the PMOF coated by a gold mir ror. In the other arm, the light is frequency shifted by an AOM, exits the PMOF, is collimated by a $50 \mathrm{~mm}$ focal length lens $(\mathrm{CL})$ and its polarization is converted by the $\mathrm{PC}$ before entering the high NA objective lens to reach the sample. Then the light is back reflected toward the $\mathrm{CL}$ and injected into the PMOF. The two path beams interfere and are detected by a phototube multiplier detector (D). Since, in SSPM, the polarization is a key parameter, in order to minimize depolarization inside the optical fibers we use PMOFs and weld all of the optical fiber components (PMOF, OFC, AOM) together. This leads to a polarization extinction ratio of $14 \mathrm{~dB}$ at the level of the PMOF output oriented toward the CL. The interferometric signal

$$
\begin{aligned}
& I_{\mathrm{int}}(x, y, z, t)=I_{\mathrm{dc}}+I_{\mathrm{ac}}=I_{1}+I_{2}(x, y, z, t) \\
& \quad+2 \sqrt{ } I_{1} I_{2}(x, y, z, t) \cos \left(2 \pi f_{m} t+\psi(x, y, z, t)\right),
\end{aligned}
$$

where $I_{1}$ (resp. $I_{2}$ ) is the optical power of the light coming from the first (resp. second) arm of the interferometer. $I_{\text {int }}$ consists of a constant component $I_{\mathrm{dc}}$ and an alternative component $I_{\mathrm{ac}}$ modulated at frequency $f_{m}$. A lock in amplifier (Signal Recovery model 7280) is used to extract from $I_{\mathrm{ac}}$, the complex value:

$$
I_{\text {SSPM }}(x, y, z, t)=2 A \sqrt{ } I_{1} I_{2}(x, y, z, t) e^{i \psi(x, y, z, t)},
$$

(in volts) at modulation frequency $f_{m}$, where $A=\mathrm{GRgS}$ is the constant power to voltage conversion coefficient $\left(A \sim 5.3 \times 10^{5} \mathrm{~V} / \mathrm{W}\right)$, defined as the product of the sensitivity $S=0.05 \mathrm{~A} / \mathrm{W}$ of the phototube multiplier (D), the gain $g=$ 50 of the detector, the resistance $R=24 \mathrm{k} \Omega$ of the current voltage converter, and the amplification gain $G=12.5 / \sqrt{ } 2$ of the lock in amplifier. A $x y z$ piezoelectric stage (nPoint) is used to scan the sample in three spatial directions. This piezo electric stage is mounted on an inverted microscope (IX71 Olympus). For a fixed $x y$ position, a $V(z)$ curve is recon structed from a $z$ scanning:

$$
V(z, t)=I_{\operatorname{SSPM}}(z, t)=2 A \sqrt{ } I_{1} I_{2}(z, t) e^{i \psi(z, t)} .
$$

Figure 2(a) shows in solid (resp. dashed) line the experimental $|V(z)|$ curves obtained for a $45 \mathrm{~nm}$ gold layer immersed in water for $\mathbf{p}$ (resp. s) polarization. The periodic oscillations vis ible for positive $z$ defocus on the right of the central peak are a signature of SPP [24,25,51]. As expected for $\mathbf{S}$ polarization, the $|V(z)|$ curve decreases monotonously for positive $z$ defocus; there are no oscillations. The complex reflectivity function $P^{2}(\theta) R(\theta)$ can be reconstructed by taking the inverse Fourier transform of the experimental $V(z)$ curve [Eq. (1)] $[42,52,53]$ [Figs 2(b) and 2(c)]. The dashed curve in Fig. 2(b) can provide quantitative information on the beam profile at the objective lens aperture if we assume that in azimuthal polari zation illumination $R_{s}(\theta)$ is nearly constant and equal to 1 and
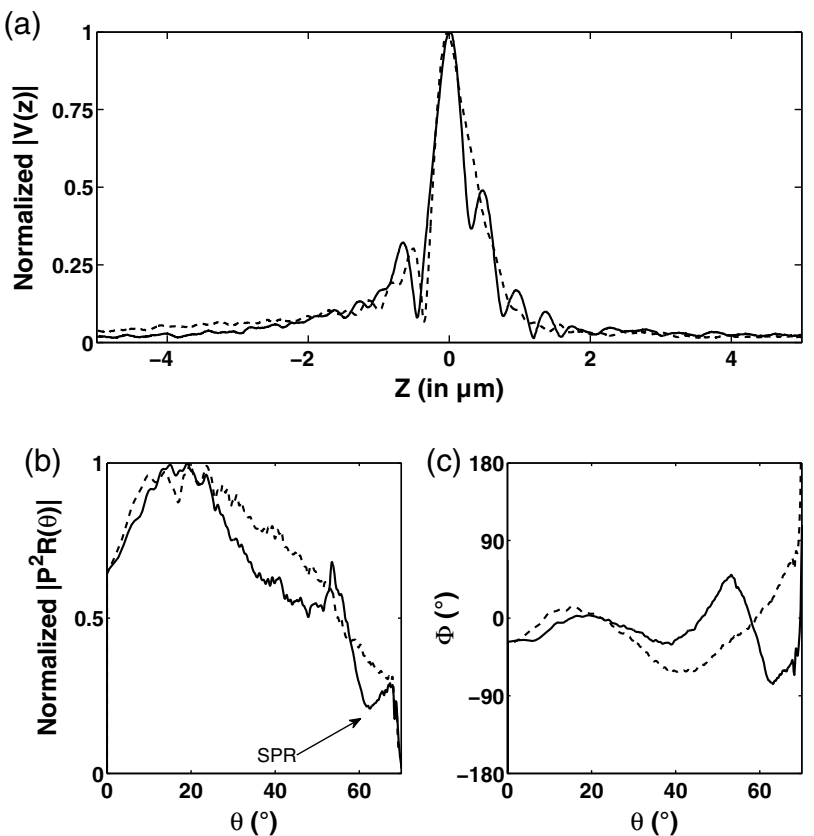

Fig. 2. (a) Experimental normalized $V(z)$ modulus curves for $\mathbf{p}$ polarization (solid line) and $\mathbf{s}$ polarization (dashed line) measured on a $45 \mathrm{~nm}$ gold layer in water. (b) and (c) represent the modulus and the phase of the reflectivity $P^{2}(\theta) R(\theta)$ versus the angle $\theta$, com puted from the experimental curves in (a), using Eq. (1) (see text). 
that the beam profile has a radial symmetry for the angle $\varphi$. $\theta=0^{\circ}$ and $\theta_{\max }=\operatorname{asin}\left(\mathrm{NA} / n_{1}\right)$ correspond to the center and the border of the objective lens aperture, respectively. We can notice that the intensity of the beam decreases from the center toward the border of the objective lens aperture. This decrease corresponds to the Gaussian beam profile of the laser beam; the illumination beam diameter at the entrance of the objective lens is $9.6 \mathrm{~mm}$, which is larger than the $6 \mathrm{~mm}$ diameter of the aperture. In the center of the beam, inside a disk with a diameter of $1.4 \mathrm{~mm}$, the intensity decreases slightly due to a liquid crystal defect at the center of the polarization con verter PC. As explained in a former work [32], the visibility of interference oscillations in the $V(z)$ curves depends on the ratio of intensities at the center and at the surface plasmon excitation angle. This ratio is nearly 2 , which ensures a good weight for surface plasmon excitation and so a good visibility of the oscil lations in the $V(z)$ curves. The SPR appears in the $\mathbf{p}$ polari zation reflectivity curve at the angle $\theta_{\mathrm{SPR}}=62^{\circ}$. This SPR angle depends on the local refractive index of the medium in contact with the gold layer. Thus, as reported in Ref. [42], changes in the refractive index can be captured by measuring the local period of the $|V(z)|$ curve for positive $z$ values. But, as for each $(x, y)$ pixel the time required for $|V(Z)|$ recording is $\sim 10 \mathrm{~s}$, it becomes quite a prohibitive task for a full $(500 \times 500)$ pixel matrix.

\section{B. Image Reconstruction and Contrast Optimization}

Imaging can be performed in a reasonable time $(\sim 50 \mathrm{~s})$ as com pared to the characteristic time of the dynamics of the object under study by fixing some $z^{*}$ position selected from the $V(z)$ curves. For a given $z^{*}$ position, a $500 \times 500$ pixel image can be recorded from $2 \mathrm{D}$ scanning along $x y$ directions. For every pixel of the image, the raw intensity $I_{R}$ is given by the modulus of $I_{\text {SSPM }}$ :

$$
I_{R}(x, y, t)=\left|I_{\text {SSPM }}\left(x, y, z^{*}, t\right)\right|=2 A \sqrt{ } I_{1} I_{2}\left(x, y, z^{*}, t\right) .
$$

Before each image acquisition, a single $V(z)$ curve is recorded at the first pixel of the image. This $V(z)$ curve is used as a reference curve to determine the exact position of the gold liquid medium interface (the value $z=0$ corresponds to the position of the $V(z)$ maximum) and to normalize the raw im age $I_{R}$ by the maximum $V(z=0)$. The normalized image $I_{N}$ is then obtained as

$$
I_{N}(x, y, t)=\frac{I_{R}(x, y, t)}{|V(z=0)|} .
$$

The contrast mechanism of SSPM imaging in a liquid medium is described by the $V(z)$ curves. When performing this imaging at a fixed scanning height $z^{*}$, the background intensity is not constant. These unwanted variations are due to some tilt be tween the $x y$ scanning plane and the sample gold surface. In other words, the effective value of $z^{*}$ is not maintained constant during $x y$ scanning. We showed previously [54] that the $z$ axis position can be adjusted in real time to keep the effective scan ning height $z^{*}$ constant. As compared to the method proposed in Ref. [54], here we will perform the tilt correction from the $|V(z)|$ curve obtained with an azimuthally polarized beam. As an illustration, we imaged in Fig. 3 a fixed C2C12 mouse my oblast cell maintained in a phosphate buffered saline solution
(PBS) (Appendix A). Note that changes in the $V(z)$ curve due to cells in an aqueous medium are expected to be smaller than in air since the refractive index drop between the cell and the culture medium is five to ten times smaller (0.03 to 0.06$)$. We recorded 200 SSPM images for $z^{*}$, varying from $2 \mu \mathrm{m}$ to $4 \mu \mathrm{m}$, and for each radial and azimuthal polarization condition. A SSPM image $I_{N}$ in radial polarization is shown in Fig. 3(a). Even if the tilt correction has been used during the $x y$ scanning, it remains a small out of plane movement that we can estimate to be around $50 \mathrm{~nm}$ over the whole image $(110 \mu \mathrm{m}$ size).

For each image $I_{N}\left(x, y, z^{*}\right)$, we have computed its proba bility density function (PDF) [black curve in Fig. 3(b)] and its cumulative distribution function (CDF) [Fig. 3(c)], from which a minimal $I_{N}^{-}$and maximal $I_{N}^{+}$normalized intensity val ues have been determined at $5 \%$ and $95 \%$ of the CDF curve. $I_{N}^{-}\left(z^{*}\right)$ and $I_{N}^{+}\left(z^{*}\right)$ are plotted in Fig. 3(d). We notice that the two curves follow a typical $|V(z)|$ profile, which means that the residual tilt after correction becomes negligible as compared to the whole dynamics of the $|V(z)|$ curves. Note also that the tilt effect does not impact locally too much the intensity variation due to the presence of the object. Consequently, we will remove a background surface $I_{B}(x, y, t)$ [Fig. 3(e)] to each image to obtain the corrected image $I_{C}(x, y)$ [Fig. 3(f)]:

$$
I_{C}(x, y, t)=I_{N}(x, y, t) \quad I_{B}(x, y, t) .
$$

For each $z^{*}$ position, this background surface $I_{B}$ is calculated by first fitting the image with a $2 \mathrm{D}$ polynomial function of or der 2 in the $x$ and $y$ directions outside the cell and then by interpolating inside the cell. In the so corrected image $I_{C}$ in
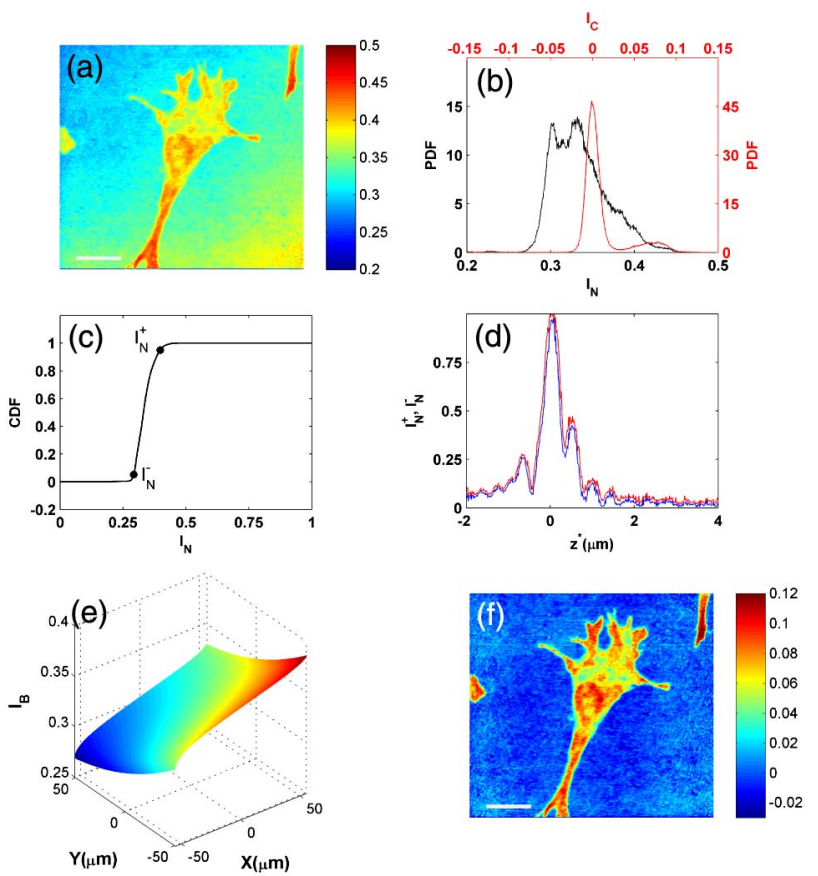

Fig. 3. (a) SSPM image $I_{N}$ of a fixed $\mathrm{C} 2 \mathrm{C} 12$ mouse myoblast cell in PBS. (b) Probability density function (PDF) of $I_{N}$ (black) and $I_{C}$ (red). (c) Cumulative distribution function (CDF) of $I_{N}$. (d) $I_{N}$ (blue curve) and $I_{N}^{+}$(red curve) versus $z^{*}$. (e) Background surface $I_{B}$ (see text). (f) Corrected SSPM image $I_{C}=I_{N} \quad I_{B}$ [Eq. (7)]. The scale bar in (a) and (d) is $20 \mu \mathrm{m}$. 
Fig. 3(f), we can observe that the cell is easily distinguishable from the background. Interestingly, the PDF of the corrected image $I_{C}$ [red curve in Fig. 3(b)] reveals the existence of a bimodal distribution as the signature of the presence of two populations that can be easily separated. The first one is cen tered at zero and corresponds to the image background (gold roughness, noise, and out of plane scanning), whereas the sec ond distribution, centered around 0.075 , is related to the cell adsorbed on the gold layer.

In Fig. 4, corrected SSPM images $I_{C}$ are shown for radially and azimuthally polarized beams at positions $z^{*}=0.39 \mu \mathrm{m}$ and $z^{*}=1.05 \mu \mathrm{m}$, respectively. The cell is far less visible with an azimuthally polarized beam [Fig. 4(b)] than with a radially polarized beam [Fig. 4(a)]. To provide a better insight into the image formation with SSPM, let us investigate the evolution of the SPPM images versus the scanning height position $z^{*}$ and the polarization illumination conditions. In Fig. 4(c), the $|V(z)|$ curves have been reconstructed from 200 SSPM images $I_{N}$ for $z^{*}$ between $2 \mu \mathrm{m}$ to $4 \mu \mathrm{m}$, at pixels A and B shown in Fig. 4(a), for radial and azimuthal polarization illuminations. The $|V(z)|$ curves for the radially polarized beam present differences between the two positions whereas for the azimu thally polarized beam the two curves are almost superimposed.

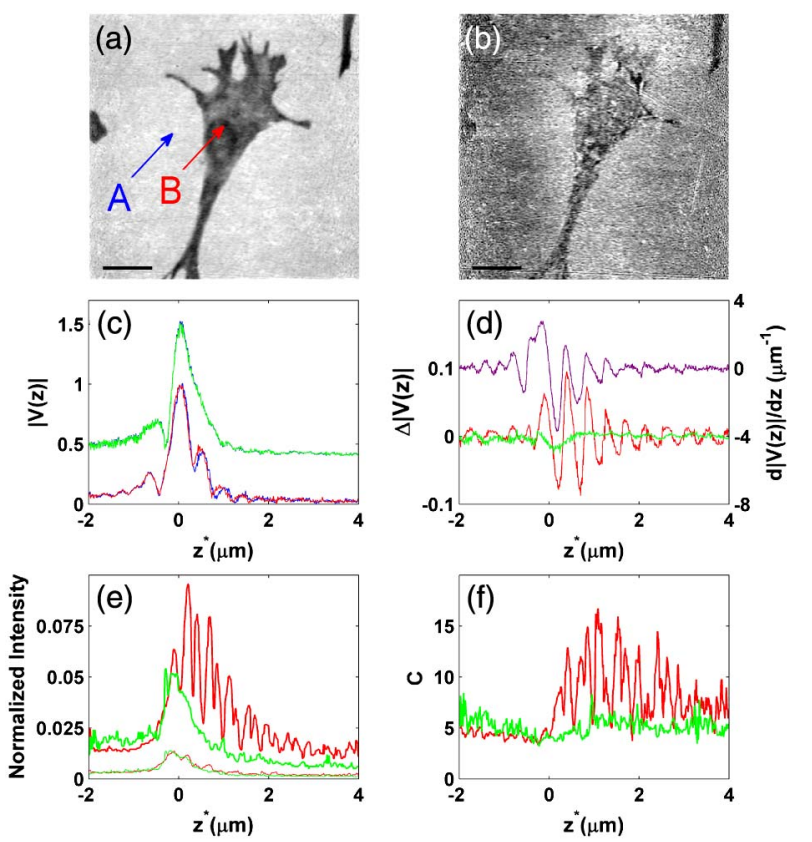

Fig. 4. (a) Corrected SSPM image $I_{C}$ of the same fixed myoblast as in Fig. 3(a) obtained for radial polarization illumination at scanning position height $z^{*}=0.39 \mu \mathrm{m}$; the intensity scale is [ $0.02 ; 0.12$ ]. (b) Same as in (a) for azimuthal polarization illumination at $z^{*}=1.05 \mu \mathrm{m}$, the intensity scale is [ 0.0060 .008 . In (a) and (b), scale bars are $20 \mu \mathrm{m}$. (c) Reconstructed $|V(z)|$ curves at pixels A (outside the cell) and B (inside the cell) from 200 images $I_{N}$, in radial polarization: A (blue) and B (red), and in azimuthal polarization: A (black) and B (green). For clarity, the $|V(z)|$ curves in azimuthal polarization are vertically shifted by 0.5 . (d) $\Delta\left|V\left(z^{*}\right)\right|$ and $\frac{d V\left(z^{*}\right)}{d z}$ (pur ple). (e) Image dynamics $\Delta I_{c}\left(z^{*}\right)$ (thick lines) and rms roughness $\sigma_{b}\left(z^{*}\right)$ (thin lines). (f) Image contrast $\mathcal{C}\left(z^{*}\right)$. In (d), (e), and (f), the red (resp. green) lines correspond to radially (resp. azimuthally) polarized beam.
The differences $\Delta|V(z)|$ between the two $V(z)$ curves, for ra dially and azimuthally polarized beams, are shown in Fig. 4(d). For radially polarized beams, $\Delta|V(z)|$ varies with $z^{*}$ and os cillates between positive and negative values for positive defocus as the signature of some shift in the local period of $|V(z)|$ caused by refractive index variations. For azimuthally polarized beams, $\Delta|V(z)|$ is by far smaller than for radial polarization and does not show any oscillations. This illustrates the ineffi ciency of azimuthal polarization to characterize refractive index changes.

Let us now extend our single pixel analysis to the entire im age $I_{C}$, and introduce the notions of image dynamics and contrast. For each image, we define its dynamics by $\Delta I_{C}=I_{C}^{+} I_{C}^{-}$, where $I_{C}^{+}$and $I_{C}^{-}$correspond to the $95 \%$ and 5\% CDF levels, respectively. As shown in Fig. 3(b), the PDF of $I_{C}$ does not follow a normal distribution and the part of the histogram related to the cell has a smaller weight and is well separated from the background at higher intensity values. This argues in favor of using the CDF instead of the PDF stan dard deviation for computing $I_{C}^{+}$and $I_{C}^{-}$. The image dynamics $\Delta I_{C}$ [Fig. 4(e)] evolves versus the scanning height position $z^{*}$ with clear oscillations for radial polarization illumination (thick red line), whereas the curve obtained for azimuthal polarization illumination (thick green line) shows a single peak around $z^{*}=$ 0 without oscillations and is always below the curve for radial polarization illumination. This underlines the amplification of the image dynamics for radially polarized beams as compared to azimuthally polarized beams. The amplification is supported by SPPs. To quantify the image contrast versus $z^{*}$ and versus the polarization mode, the image dynamics $\Delta I_{C}$ must be compared to the background noise level for each image. The limit of de tection of the SSPM in liquid is not ruled by the electronic noise (which is 0.0018 and can be lowered to 0.0009 if the frequency modulation $f_{m}$ is increased from 140 to $1200 \mathrm{kHz}$ ), but by the background signal, mainly due to the gold rough ness. The standard deviation $\sigma_{b}$ of a bare gold area of the image $I_{C}$ is computed for every $z^{*}$ and is represented in Fig. 4(e) by a red (resp. green) thin line for radial (resp. azimuthal) polariza tion illumination. The background noise for both polarization conditions is $z^{*}$ dependent since it is maximal near $z^{*}=0$ and decreases with the defocalization $z^{*}$. We define the image con trast $\mathcal{C}$ as the ratio between the image dynamics $\Delta I_{C}$ and the background noise standard deviation $\sigma_{b}$ for each scanning height position $z^{*}$ :

$$
\mathcal{C}\left(z^{*}\right)=\Delta I_{C}\left(z^{*}\right) / \sigma_{b}\left(z^{*}\right) \text {. }
$$

$\mathcal{C}\left(z^{*}\right)$ is shown in Fig. 4(f) for radially polarized (thick red line) and azimuthally polarized (thick green line) beams. The former $\mathcal{C}\left(z^{*}\right)$ displays oscillations and values higher than 11 for a wide range of $z^{*}$ values (between 0.39 and $2.9 \mu \mathrm{m}$ ). The latter $\mathcal{C}\left(z^{*}\right)$ is lower and much flatter and almost independent of $z^{*}$. These results show that SPPs are efficient in contrast amplification for $z^{*}$ between 0.39 and $2.9 \mu \mathrm{m}$. Between $z^{*}=0$ and $0.39 \mu \mathrm{m}$ and above $2.9 \mu \mathrm{m}$, the image contrast produced by SPPs is hardly distinguishable from that obtained without SPPs. Interestingly, the $z^{*}$ positions where $\mathcal{C}\left(z^{*}\right)$ is locally maximum correspond to the extrema of $\Delta|V(z)|$ [red curves in Figs. 4(d) and $4(\mathrm{f})$ ], which confirms that the contrast is strongly depen dent on the shape of $|V(z)|$. However, the highest contrast 
value is obtained for $z^{*}=1.12 \mu \mathrm{m}$ rather than for $z^{*}=0.22 \mu \mathrm{m}$, as expected from $\Delta\left|V\left(z^{*}\right)\right|$ and the image dynamics $\Delta I_{C}\left(z^{*}\right)$ curve in Figs. $4(\mathrm{~d})$ and $4(\mathrm{e})$.

Experimentally, we need to select the value of $z^{*}$ to perform the image $x y$ scanning. In Fig. 4(f), we observe that the contrast $\mathcal{C}\left(z^{*}\right)$ is strongly oscillating in the 0.39 and $2.9 \mu \mathrm{m} z^{*}$ range, so that a $100 \mathrm{~nm}$ drift in $z^{*}$ may dramatically decrease the con trast. We have also noticed that the positions for which the image contrast is locally maximum fluctuate from sample to sample over a range of $200 \mathrm{~nm}$. These variations may be attrib uted to fluctuations in gold and coverslip thicknesses. Actually, thickness changes of the gold layer mainly entail modifications of the $|V(z)|$ oscillation amplitude, whereas mismatches be tween the real and optimal coverslip thicknesses introduce phase aberrations that shift the $|V(z)|$ oscillation positions. Since drawing similar $\Delta V\left(z^{*}\right)$ or $\mathcal{C}\left(z^{*}\right)$ curves for each sample is prohibitively time consuming, a simpler and faster way to guess $z^{*}$ positions with maximal contrast is to use the derivative $\frac{d|V(z)|}{d z}$ computed from one single $|V(z)|$ curve. As shown in Fig. 4(d), the $\Delta|V(z)|$ oscillations are strongly correlated to those observed in $\frac{d|V(z)|}{d z}$; the extrema fall at the same $z^{*}$ posi tions within an error of $\sim 30 \mathrm{~nm}$. Note that this comparison is possible because the local RI changes induce very little varia tions in the $|V(z)|$ curves.

\section{Sensitivity and Limit of Detection}

To complete our discussion of the choice of $z^{*}$ for optimal con trast, we must also estimate the sensitivity $S_{\text {SSPM }}$ and the de tection limit in RI units (RIU) of the microscope at this position $z^{*}$ when the refractive index $n$ of the medium changes. The sensitivity $S_{\text {SSPM }}$ can be computed from the value of $\left|V\left(z_{m}\right)\right|$ taken at the position $z_{m}$ corresponding to a maxima of $\frac{d\left|V\left(z_{m}\right)\right|}{d z}$ :

$$
\mathcal{S}_{\text {SSPM }}=\frac{\delta\left|V\left(z_{m}\right)\right|}{\delta n} .
$$

Using a 4 layer model (Appendix C), the theoretical value $S_{\text {SSPM }}^{\text {theo }}$ was estimated at $\sim 3.3$ for RI drops in the interval $\left[10^{-4}, 6.10^{-2}\right]$. The experimental $S_{\text {SSPM }}^{\exp }$ was measured from mixtures of water and glycerol, with RIs calibrated with a refractometer, giving RI drops from $8.7 \times 10^{-3}$ to $5.3 \times 10^{-2}$. The single point limit of detection of the SSPM microscope is actually given by the footprint $\left|V_{s n}(z)\right|$ of the shot noise on the $V(Z)$ curves. In heterodyne interferometry, the signal to shot noise ratio $|V(z)| /\left|V_{\text {sn }}(z)\right|$ is written as the ratio of the variance of the detector current $\left\langle i_{\text {ac }}^{2}\right\rangle_{t}$ over the vari ance of the shot noise current $\left\langle i_{\mathrm{sn}}^{2}\right\rangle_{t}$

$$
\frac{|V(z)|}{\left|V_{\mathrm{sn}}(z)\right|}=\sqrt{\frac{\left\langle i_{\mathrm{ac}}^{2}\right\rangle_{t}}{\left\langle i_{\mathrm{sn}}^{2}\right\rangle_{t}}}=\sqrt{\frac{2 \tau S}{e}} \frac{I_{1} I_{2}}{I_{1}+I_{2}} \simeq \sqrt{\frac{2 \tau S}{e}} I_{2},
$$

where $e$ is the electron charge and $\tau$ is the integration time of the lock in amplifier $(\tau=100 \mu \mathrm{s})$. For our experimental setup at $z_{m}=1.12 \mu \mathrm{m}$, the optical power $I_{2}=I_{1} / 270=50 \mathrm{nW}$ leads to $|V(z)| /\left|V_{\text {sn }}(z)\right| \sim 1.8 \times 10^{3}$. Hence, a shot noise level $\left|V_{\mathrm{sn}}(z)\right|=8.33 \times 10^{-5}$ on $V(z)$ curves gives a limit of detec tion of $\sim 5.6 \times 10^{-5}$ RIU. This theoretical limit could be further improved by a factor of 30 by (i) replacing the $50 \mathrm{~mm}$ focal length lens by a shorter focal length lens system and adding a beam expander system for a better light coupling and (ii) replacing the photomultiplier tube detector by a photodiode (the quantum efficiency and so $S$ could be enhanced by seven). Such modifications would lead to an ultimate theoretical limit of detection of $1.8 \times 10^{-6}$ RIU for this SSPM technique.

In imaging mode, additional sources of noise are involved that lower the RI limit of detection. The gold layer roughness generates a background noise signal $\sigma_{b}$ estimated at $\sim 0.0035$, from the thin red curve on Fig. $4(\mathrm{e})$ at $z_{m}=1.12 \mu \mathrm{m}$. This value is identical to that obtained from a bare gold surface in water, meaning that cellular fragments that could deposit on the gold surface do not change drastically the SSPM signal fluctuations. From $S_{S S P M}^{\exp }$, we compute the RI limit of detec tion: $\Delta n_{\lim }=\sigma_{b} / S_{\text {SSPM }}^{\text {exp }} \sim 2.3 \times 10^{-3}$ RIU. Loison and Fort [55] estimated a detection limit of $4 \times 10^{-4}$ RIU, which can be concluded as a six fold improvement. However, the RI drop detection limit is not independent of the thickness and the area of the considered dielectric layer, since the HRSPM microscope is sensing a whole volume in the evanescent field of the gold/dielectric interface. For comparing different experimental works, we thus need to compute $V_{I}=h \mathcal{A} \Delta n$, where $h$ is the thickness of the dielectric layer and $\mathcal{A}$ is the area of the sensed region (proportional to the PSF of the microscope). For a PSF of $181 \mathrm{~nm}$ (see Section 2.D), with the same 4 layer model as above (Appendix C), we get $\sigma_{b}=0.0035$, leading to a volume detection limit of $\sim 4720 \mathrm{~nm}^{3}$. In comparison, the volume de tection limit obtained in Ref. [55] 15000 $\mathrm{nm}^{3}$ is much less impressive. In the same line, Watanabe et al., [35] used micro patterned phospholipid bilayers with a $4 \mu \mathrm{m}$ diameter to con clude that their localized surface plasmon has a thickness res olution of $0.33 \mathrm{~nm}$ for an index drop of 0.113 , which would give a volume detection limit of $1080 \mathrm{~nm}^{3}$, which is four times smaller than what the SSPM device described here can afford. However, it must be noted that the computations in Ref. [35] were limited to small (micron size) structures with well defined bilipidic layers and did not take into account the background noise on large surface areas (as we do here). Finally, the HRSPM techniques in Refs [35,55] are only sensitive to SPR angle shift, whereas SSPM is also sensitive to SPR angle spread ing coming from radiative leakage. Since gold layer roughness (i.e., local submicronic gold thickness variations) induces radi ative leakage, the SSPM background noise is amplified and the RI detection limit is increased. Given that the SSPM back ground fluctuations can be scaled down by a factor of 3 to 4 by a surface treatment of the glass coverslip prior to gold sput tering [56], SSPM could nevertheless reach the same volume detection limit as other HRSPMs.

\section{Stability for Time-lapse Imaging}

The use of an interferometer automatically raises the issue of stability and reproducibility of the optical signal and conse quently of the stability and reproducibility in SSPM imaging. There are four bottlenecks that should be discussed: (i) incor rect light retro injection through the CL inside the PMOF, (ii) errors in the distance $z^{*}$ given by mechanical and thermal drifts of the sample, the objective lens and the microscope stand, (iii) out of plane movements produced by the $x y z$ 
piezoelectric stage in the $z$ direction and positioning errors in $x y$ directions, (iv) spurious reflected light that perturbs the interference signal $I_{\mathrm{ac}}$. In this section, we discuss the stability of the SSPM with tentative estimation of the respective weights of these four kinds of instability.

Concerning thermal drift [bottleneck (ii)], an autofocus is performed at the beginning of each image, since the recorded $|V(z)|$ curve provides the exact position of the gold surface. The autofocus compensates drastically the thermal and mechanical drifts of the setup in the $z$ direction. Actually, we have estimated a root mean square drift equal to $9.4 \mathrm{~nm}$ in $z$ positioning for 27,183 recorded images ( $491 \mathrm{~h}$ recording). If we convert this spatial drift in terms of intensity $I_{N}$ by multi plying the drift by the derivative of $|V(z)|$, we get an intensity drift ranging between 0.012 and 0.0188 . The influence of in stabilities of types (i) and (iv) can be studied from the repro ducibility of $|V(z)|$ curves at one $x y$ position on the surface. The instability (iv), due to spurious reflected light, can be com puted from the $|V(z)|$ curves since it appears as a very slowly varying function of $z$ on the real and imaginary parts of $I_{\text {SSPM }}(x, y, z, t)$ [Eq. (3)]. The fluctuation standard deviation due to spurious reflected light, when estimated over $175 \mathrm{~min}$ $(100|V(z)|$ curves), is 0.025 . After elimination of these fluc tuations, the remaining $|V(z)|$ fluctuations are smaller than 0.015 . From the $5 \mathrm{~nm}$ accuracy in the $z$ positioning, we esti mate the contribution to $|V(z)|$ of the type (iii) instability to be $0.0075 \pm 0.0025$. The fluctuations due to the type (i) insta bility, i.e., light retro injection, produce an error of $0.0075 \pm$ 0.0025 in $|V(z)|$. If these fluctuations are compared to the $I_{N}$ value at $z^{*} \sim 0.4 \mu \mathrm{m}$, they represent for instabilities i), ii), and iv) no more than $1.8,1.8$, and $6.3 \%$, respectively. Consequently, the total fluctuations $\sim 9.9 \%$ comes mainly from a spurious reflected light instability.

We have tested the overall imaging stability and reproduc ibility on 180 images of the fixed myoblast cell of Fig. 4, recorded for the same scanning height position $z^{*}=0.39 \mu \mathrm{m}$ (195 min). When considering the normalized images $I_{N}(x, y)$, we find that for each pixel of the image the temporal standard deviation of $\sigma_{I_{N}}(x, y)$ ranges between 0.019 and 0.0309 , i.e., between 5 and $10 \%$ of the whole image time averaged normal ized intensity $\left\langle I_{N}\right\rangle_{t}$. If we consider now the corrected image $I_{C}$, we get from the 180 images that inside the cell, the time stan dard deviation of $\sigma_{I_{C}}(x, y)$ is $\sim 0.006$, as shown in Figs. 5(c) and 5(d) (the borders of the cells were not taken into account because of a drift in $x y$ direction between images). This means that the errors induced by the instability of the interferometers are, in the worst case, at the same level as the background noise $\sigma_{b}$ of the image (which is equal to 0.0069 at $z^{*}=0.39 \mu \mathrm{m}$ ). As shown in Figs. 5(e) and 5(f), the signal to noise ratio $\operatorname{SNR}(x, y)=\left\langle I_{C}(x, y)\right\rangle_{t} / \sigma_{I_{C}}(x, y)$ (where $\left\langle I_{C}(x, y)\right\rangle_{t}$ is the time average of images $I_{C}$ ) inside the cell is as large as 10 to 30 . Then, by inverting the SNR, we get that $\sigma_{I_{C}}$ inside the cell is between 3.5 and $10 \%$ of the time averaged image intensity $\left\langle I_{C}\right\rangle_{t}$. This fluctuation estimate seems to be lower than the one extracted from the $V(z)$ curves. In fact, a signifi cant amount of the fluctuations has been removed when per forming the background substaction to get the corrected intensity $I_{C}$ [Eq. (7)]. This measurement of stability on fixed
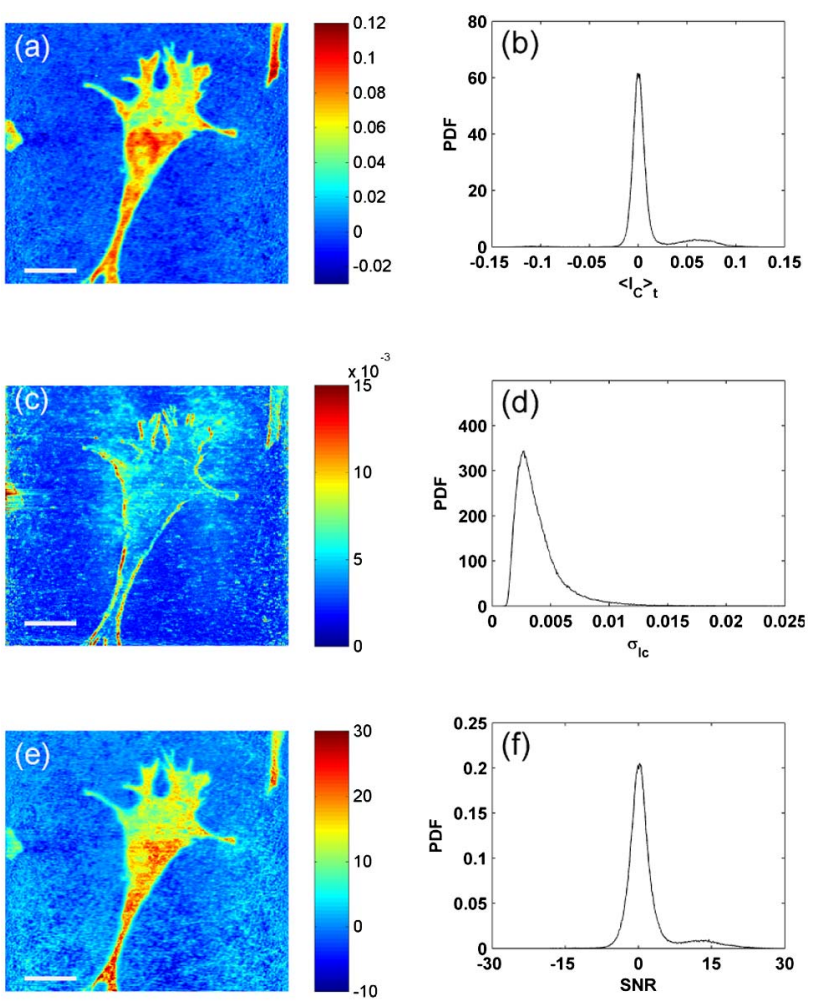

Fig. 5. (a) Time averaged $\left\langle I_{C}(x, y)\right\rangle_{t}$, over 180 images of a fixed myoblast cell, recorded over $195 \mathrm{~min}$ at $z^{*}=0.39 \mu \mathrm{m}$. (c) Corresponding standard deviation $\sigma_{I_{C}}(x, y)$. (e) Signal to noise ra tio $\operatorname{SNR}(x, y)=\left\langle I_{C}(x, y)\right\rangle_{t} / \sigma_{I_{C}}(x, y)$. (b), (d), and (f) correspond to the PDF of pixel values of the images shown in (a), (c), and (e), respectively.

cells confirms that the SSPM images are stable over a long period of time. This is the demonstration that the microscope with the heterodyne optical fiber interferometer provides a good stability. Another main advantage due the optical fiber design is the drastic minimization of optical alignment opera tions. For this microscope set up, no optical alignment or ad justment was needed for a period of 22 months, even if the microscope was used on a daily basis. Except for the coverslip, nothing else was changed on the microscope setup during this period. In summary, the stability of the heterodyne optical fiber interferometer opens the way for time lapse imaging of the dy namics of living cells.

\section{E. Resolution and Penetration Depth}

As shown in Fig. 4(a), the contour of the fixed myoblast cell is well resolved with submicronic resolution. As measured with a $50 \mathrm{~nm}$ gold particle, the experimental point spread function (PSF) of the SSPM in aqueous medium in radial polarization has a full width at half maximum (FWHM) of $\sim 180 \mathrm{~nm}$ in $x$ and $y$ directions (Fig. 6). We conclude that the SSPM spatial resolution is limited by diffraction and is quite consistent with the theoretical value of $150 \mathrm{~nm}$, obtained by computing the electromagnetic field near the focus of a radially polarized beam illuminating a $45 \mathrm{~nm}$ gold layer immersed in water (data not shown). The small difference between the experimental and 
(a)
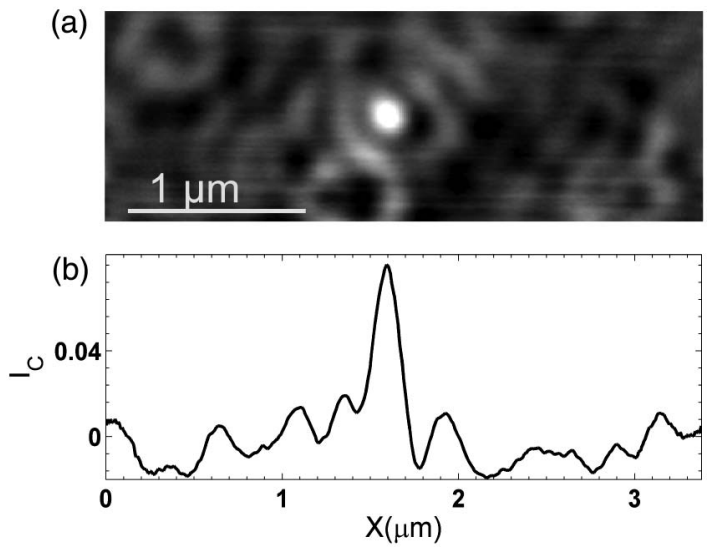

Fig. 6. (a) SSPM image $I_{C}$ with a radial polarization illumination at $z^{*}=0.425 \mu \mathrm{m}$ of a $50 \mathrm{~nm}$ gold particle in water. The intensity scale is [ $0.02 ; 0.06]$. (b) Intensity profile $I_{C}$ along a horizontal section crossing the particle in its center. The FWHM of the central peak is $181 \mathrm{~nm}$.

theoretical values is certainly due to the aberrations introduced by the thickness mismatch of the coverslip.

SPPs probe the cell region that is inside the evanescent field. In the microscope, the image reconstruction is performed at a defocused position $\left(z^{*}>0\right)$, making the relation between the region probed by the SSPM and the SPP penetration depth not immediate, since the cell modifies locally the evanescent field penetration length. To estimate the depth of the probed region with the SSPM relative to the SPP penetration depth, we have imaged a $10 \mu \mathrm{m}$ polymer sphere (polymethylsilsesquioxan Kobo diasphere KS 1000 sphere), with the SSPM at different positions $z^{*}$. Imaging has been performed in an ethanol/water (50/50\%) medium in order to lower the RI drop between the object $(n=1.41)$ and the immersion medium $(n=1.362)$ to the typical RI drop of biological cells in their culture medium. The area probed with the SSPM [Figs. 7(b) and 7(d)] is by far smaller than the sphere size [Fig. 7(a)]. When moving away from the center of the particle, the sphere is no longer in con tact with the surface and its separation distance increases [Fig. 7(c)]. The lateral position $X_{p}$ where the particle is no longer detected with the SSPM provides an estimate of the depth of penetration $d_{p}=R \quad \sqrt{R^{2}} \quad X_{p}^{2}$, where $R$ is the sphere radius [Fig. 7(c)]. To determine $X_{p}$ from the SSPM in tensity profile of the particle, two criteria can be used: $X_{p}$ is estimated when the normalized intensity of the image (i) is di vided by a factor $e$ or (ii) equal to the background noise stan dard deviation $\sigma_{b}$. As shown in Fig. 7(e), $d_{p}$ evaluated using either criteria definitely increases as the defocus position $z^{*}$ in creases (from 100 to $300 \mathrm{~nm}$ for the former and from 180 to $600 \mathrm{~nm}$ for the latter). This is another difference from other TIRF based HRSPM [45] or scanning HRSPM [35]. In SSPM, the electromagnetic field at the interface is not a simple evanescent wave; part of the laser beam that does not undergo total reflection generates a focused beam above the gold surface whose position moves away from the gold interface when in creasing the height position $z^{*}$. This explains the increase of $d_{p}$ with $z^{*}$ reported in Fig. 7 (c). In the following application to
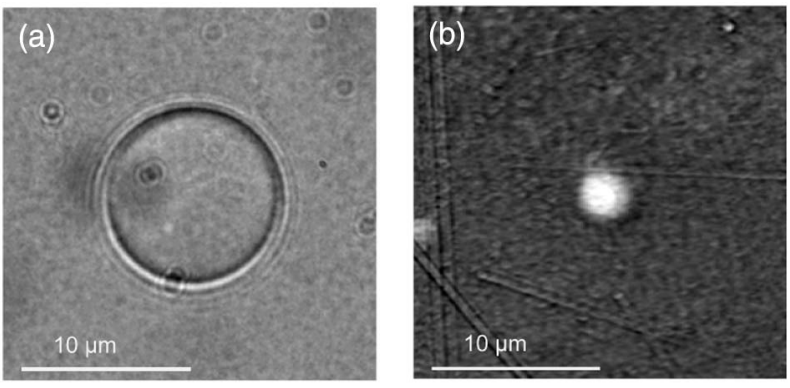

(c)
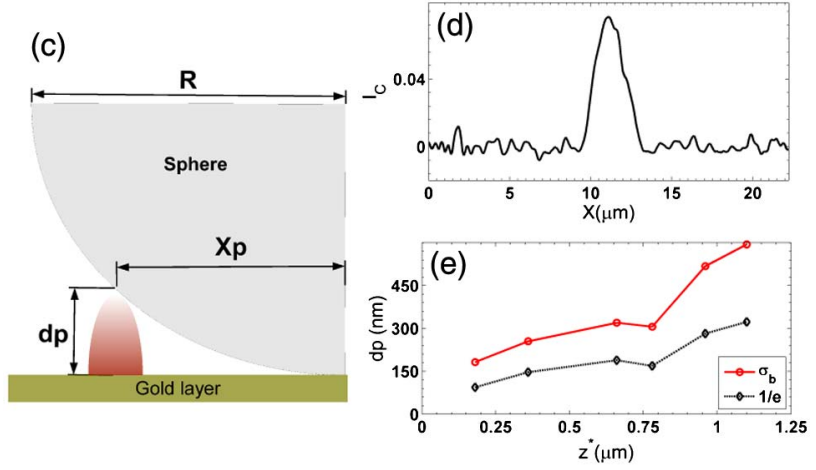

Fig. 7. Penetration depth of the SSPM probed with a $10 \mu \mathrm{m}$ poly mer bead immersed in a 50\%/50\% ethanol water medium probed by SSPM. (a) Transmission microscopy image. (b) SSPM image $I_{C}$ with a radially polarized beam at scanning height position $z^{*}=0.36 \mu \mathrm{m}$ of the same bead. (c) Sketch of the field probed by the SSPM. (d) Intensity profile $I_{C}$ along a horizontal section crossing the particle in its center. (e) Evolution of the penetration depth $d_{p}$ versus the scan ning height position $z^{*}$ using criteria (i) (black) and (ii) (red) (see text).

living cell imaging, we have chosen $z^{*}$ at the first maximum of the derivative of $V(z)$ [Fig. 4(b)], between 350 and $500 \mathrm{~nm}$ corresponding to a penetration depth between 250 and $290 \mathrm{~nm}$. We have made this choice in order to get the best contrast and to restrain the probed domain of biological cells only to the near field of SPPs.

\section{LIVING CELL IMAGING}

As a first application of SSPM to living cell imaging in liquid, we use again the $\mathrm{C} 2 \mathrm{C} 12$ myoblast cell model under the cell culture and sample preparation conditions described in Appendix A. To get the best contrast, and to restrain the cell probed domain to the near field of SSPs, we will fix $z^{*}$ at $0.4 \mu \mathrm{m}$, the first maximum of the derivative of $V(z)$ [Fig. 4(b)]. The living cells were observed with the SSPM at room temperature $\left(24^{\circ} \mathrm{C}\right)$ dur ing $50 \mathrm{~h}$, with a time interval of $65 \mathrm{~s}$.

\section{A. SSPM Cell Imaging}

From the time lapse video Visualization 1 the inter and intra cellular dynamics are noteworthy. During the $50 \mathrm{~h}$ recording of the same zone of the coverslip, cellular migration, intercellular contacts, lamellipodial and filopodial dynamics, adhesion, detachment and attachment on the gold substrate are observ able. In Figs. 8(a) 8(c) and 8(d) 8(f), two sequences of cell mi gration are reported, showing that different cells migrate over the same recorded area during $50 \mathrm{~h}$. SSPM images show different 

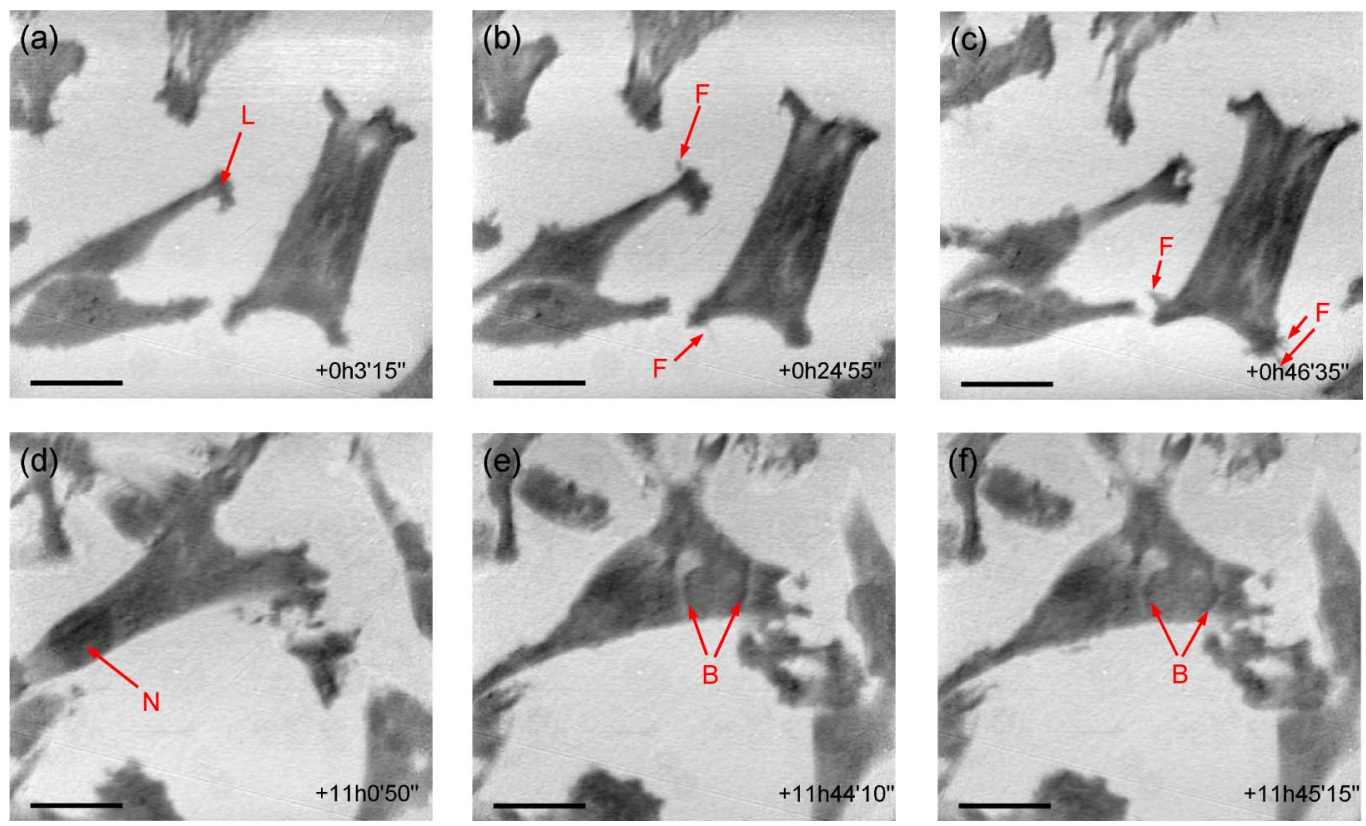

Fig. 8. SSPM images and time lapse video (Visualization 1) of living C2C12 cells during $50 \mathrm{~h}$ at scanning height position $z^{*}=0.40 \mu \mathrm{m}$. The scalar bar in all images is $20 \mu \mathrm{m}$ and the intensity scale is [ $0.02 ; 0.1]$. The arrows have the following meaning: filopodia (F), lamellipodia (L), nucleus $(\mathrm{N})$ and fronts $(\mathrm{B})$.

structures as filopodia [F arrows in (b) and (c)] and lamellipodia [L arrows in (a)]. The filopodia, which are few micrometers wide or less, are very dynamic; they explore the surface within a few micrometers distance and the same filopodia is not observable in more than two successive images $(\Delta t \sim 65 \mathrm{~s})$, giving a pro trusion retraction velocity larger than $3 \mu \mathrm{m} / \mathrm{s}$. Lamellipodia at cell extremities explore the gold surface, but also the neighbor ing cells; they show expansion and retraction during variable periods of time from minutes to more than an hour. During the time lapse video Visualization 1, we can see that the whole cell bodies migrate, for example, the central cell in Fig. 8(d) moves from left to right in Fig. 8(f). Inside cells, very intense fronts with crescent forms propagate across the cells [B arrows in Figs. 8(e) and 8(f)] during rather short periods of time (a few tens of minutes). These propagating fronts of locally higher RI volume correspond to either some parts of the cells that are denser near the surface or to some parts of the cells that are closer to the surface. In both interpretations, these propagating waves are likely to correspond to some actin network reorgani zation. Although the probed region inside the cells does not exceed $290 \mathrm{~nm}$, the nucleus sometimes approaches close to the surface $[\mathrm{N}$ arrow in Fig. 8(d)] and stays in the evanescent field during cell migration (in Visualization 1, it can be noticed for cells migrating between $+10 \mathrm{~h}$ and $+17 \mathrm{~h}$, between $+31 \mathrm{~h}$ and $+37 \mathrm{~h}$, and between $+47 \mathrm{~h}$ and $+50 \mathrm{~h})$. We attribute this dark part to the nucleus because this rather cir cular object has, as expected, a higher RI ( 1.39) than the sur rounding cytoplasm $(\sim 1.36)$. Note that when the nuclei are visible, they have the characteristic size of $\mathrm{C} 2 \mathrm{C} 12$ myoblast nu clei, as observed with DIC $(20 \pm 4 \mu \mathrm{m} \times 16 \pm 3 \mu \mathrm{m})$, and blinking spots of submicrometer size appear ahead of the nuclei in the cell migration direction as the possible indication of focal adhesion sites [57].
If we compare SSPM images with former HRSPM images of fixed or living cells in a liquid medium ([43 49]), we can em phasize some drastic differences. Peterson et al., [45] were the only ones to pinpoint focal adhesion sites (for fixed vascular smooth muscle cells A10 and human carcinoma cells HepG2) and extracellular materials (for fixed adenocarcinomic alveolar basal epithelial cells A549). Moh et al. [43] observed higher contrast domains corresponding to nuclei in fixed mouse fibro blast 3T3 cells. Jamil's et al. [46] were the first to reach the SSPM resolution on single filopodia of living cells. Since the cell substratum interaction depends on the cell type and the adhesion conditions (kind of surface, culture medium, and cell cycle phase), further tests on various cells and culture medium conditions should bring more insight on the cell substratum adhesion with HRSPM.

\section{B. Adherence Evolution}

During the $50 \mathrm{~h}$ recording, cells detaching or adhering to the surface can be dynamically observed in Visualization 1. If we compare two images, one at the beginning [Fig. 9(a)] and the other one at the end [Fig. 9(b)] of the experiment, the image contrast is quite different, with a much lower proportion of bright regions in the latter than in the former. We have computed the normalized PDF of the two images [Figs. 9(c) and 9(d)]. These histograms have a bimodal shape, with a first bump corresponding to the background noise and a second bump corresponding to the cells. These histograms can be fit ted by the sum of two Gaussian distributions of parameters $A_{i}, I_{i}, \sigma_{i}$, with $i=1,2$, for each bump [green curves in Figs. 9(c) and 9(d)]. $A_{i}, I_{i}, \sigma_{i}$ are, respectively, the maximum amplitude, the mean value, and the standard deviation of each Gaussian curve. When comparing the rightmost bump corre sponding to cell population, we notice a significant decrease of 


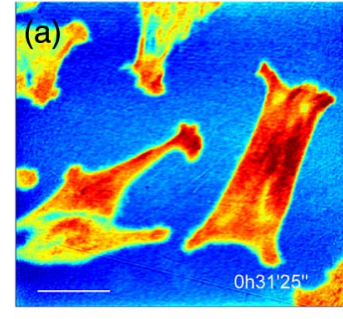

(c)
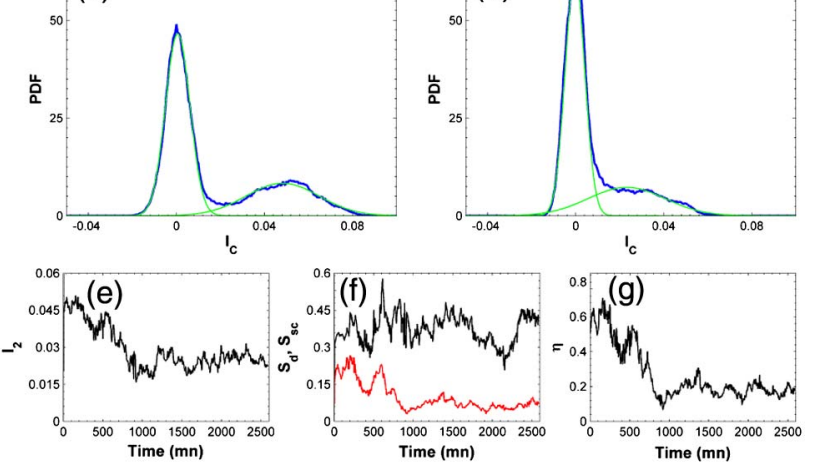

Fig. 9. Evolution in time of living cell adhesion. SSPM images: (a) at the beginning of Visualization 1 and (b) $36.5 \mathrm{~h}$ later at the end of recording. The scale bar is $20 \mu \mathrm{m}$ and the intensity scale is [ $0.02 ; 0.08]$. (c) and (d) are the corresponding PDF of images (a) and (b). Fits with a bimodal Gaussian distribution are plotted in green. (e) Corrected intensity $I_{2}(t)$ inside the cells versus time. (f) The global surface $S_{d}(t)$ covered by cells and probed by the SSPM (black) and the contact surface $S_{\text {sc }}(t)$ (red) versus time. (g) $\eta(t)=S_{\mathrm{sc}}(t) / S_{d}(t)$ (see text).

$I_{2}$ from the beginning to the end of the time lapse recording, from 0.048 to 0.025 , with a remarkable transition around 1000 min [Fig. 9(e)]. As probed by the SSPM, the global sur face ratio covered by cells, $S_{d}=A_{2} \sigma_{2} /\left(A_{1} \sigma_{1}+A_{2} \sigma_{2}\right)$ is more or less $40 \%$ and this value does not change much on average within the $50 \mathrm{~h}$ recording [Fig. 9(f)]. The decrease of $I_{2}$ with out a significant change of $S_{d}$ is an indication of some evolution in cell adhesion. Actually, the cells are in strong contact with the surface at the beginning of the recording and lose progressively this tight contact with time.

The surface proportion $S_{\text {sc }}$ of the cells that are in tight con tact with gold can be quantified. We delimit tight contact zones where the SSPM intensity is higher than half the maximum: $S_{\text {sc }}=\left\{(x, y) / I_{C}(x, y)>\max \left(I_{C}\right) / 2\right\} / S_{\text {tot }}$, where $S_{\text {tot }}$ is the total surface of the image. Two kinds of cell surface interaction fulfill this condition. First, cells that do not touch the surface but are close enough so that their bodies intersect the evanes cent field [similar to the configuration in Fig. 7(c)]. This case is observed for rather spherical cells that are $60 \mathrm{~nm}$ or less away from the gold surface. Second, rather spreaded cells with rather thick parts $(>60 \mathrm{~nm}$ ) of their body that touch (tight contact) the gold surface. $S_{\mathrm{sc}}$ is plotted in red in Fig. 9(f): at the begin ning of the recording, cells in strong contact cover about $25 \%$ of the image surface and this value decreases down to $\sim 510 \%$ at the end of the experiment, with a transition at around $1000 \mathrm{~min}$. Let us define $\eta=S_{\text {sc }} / S_{d}$, the ratio of the cell surface in strong contact $S_{\text {sc }}$ and the cell surface detected by the SSPM.
As shown in Fig. $7(\mathrm{~g})$, this ratio is important $(60 \%)$ at the be ginning but starts decreasing rather fast to reach $\sim 20 \%$ after $\sim 1000$ min ( 3 fold decrease) and then stabilizes at the end of the recording. This modification of $\eta$ points out a change in the cell adhesion, with a rather sharp transition at $1000 \mathrm{~min}$. This change in adhesion comes along with some decrease of the number of observed filopodia and their almost total disappearance after $\sim 1000$ min (see Visualization 1).

This transition is certainly related to a modification of the experimental conditions inside the chamber. Since the chamber is sealed, we suspect that the $\mathrm{pH}$ value of the DMEM culture medium has decreased because of lack of $\mathrm{CO}_{2}$ maintenance around $10 \%$. Since cellular respiration produces carbon dioxide and lactate that can acidify the culture medium over time, this may significantly affect the microenvironment of the cells due to the limited volume of growth medium inside our sealed chambers $(25 \mu \mathrm{l})$ [58]. This decrease of the culture medium $\mathrm{pH}$ might impact the intracellular $\mathrm{pH}$ and in turn limit the adhesion maturation by focal adhesion kinase (FAK) pathway [59]. Also, the amount of glucose may have decreased during the video recording, inducing a modification of the cell meta bolic synthesis of ATP, resulting in a modification of the mor phology, adhesion, and motility of the cells. Finally, during the cell cycle, the cells are synthesizing ECM proteins, cytokines, and cellular fragments that likely absorb on gold to form some hybrid layers with different adherence properties for the cells.

\section{CONCLUSION}

We report, to the best of our knowledge, the first high resolution surface plasmon imaging of living cells in a culture medium over long periods of time ( $50 \mathrm{~h}$ ) that combines SSPM and a fibered interferometer. This SSPM does not suffer from aberrations and resolution loss faced by classical wide field $\mathrm{mi}$ croscopes. We have shown that the SSPM setup is suited for capturing the tightness of cell substrate contacts in real time and their detachment as well as their anchoring. It is also suited to characterize the local fluctuations of filopodia and lamellipo dia protrusion shapes and velocities [60]. Besides its ability to capture the migration of adherent cells (few $\mu \mathrm{m} /$ minute), it can be used to compare cells with different strengths of adhesion in order to better understand their interactions with their ECM. Note that by replacing the piezoelectric stage by a motorized stage, a much wider area could be scanned, allowing us to fol low long distance cell migration and interaction and to increase the size of the cell statistical sample on a single experiment. Even if scanning methods are limited for studying fast dynam ics (faster than minutes), recent technical developments in beam focusing engineering [61] could be a future opportunity for improving the SSPM image resolution. Another possible improvement could be to couple SSPM with DIC and/or fluo rescence microscopy in order to address deeper biological issues. In particular, SSPM could straightforwardly be extended to real time high resolution imaging of cell cell interactions in epithelial layers, since it was recently demonstrated by prism coupled SPR $[62,63]$ that these layers likely play the role of wave guides. Indeed, as shown in a previous work [53], SSPM can perform submicron resolution imaging of wave guide 
defects. This opens a new field of applications of this HRSPM method to thin tissue and multilayer characterization.

\section{APPENDIX A}

\section{CELL CULTURE}

C2C12 mouse myoblast cell line (American Type Culture Collection, Manassas, VA, number CRL 1772) was cultured in high glucose $(4,5 \mathrm{~g} / \mathrm{L})$ Dulbecco's Modified Eagle Medium (DMEM, PAA, GE Healthcare Life Science, Munich, Germany) supplemented with $20 \%$ fetal bovine serum (FBS, PAA), $1 \%$ of penicillin streptomycin $(10,000 \mathrm{U} / \mathrm{mL}$, Gibco) and $10 \mathrm{mM}$ HEPES. Myoblasts were kept in a $5 \% \mathrm{CO}_{2}$ atmos phere at $37^{\circ} \mathrm{C}$ on $90 \mathrm{~mm}$ petri dishes and fresh culture medium was replaced every 2 days. At $70 \%$ of confluence to avoid differ entiation, dishes were washed twice with PBS and cells were dissociated from substrate using $0.25 \%$ trypsin EDTA (Gibco) for $3 \mathrm{~min}$ at $37^{\circ} \mathrm{C}$. Then cells were replated at a lower concen tration (30\% of confluence). Fresh culture medium was re placed each 23 days. Cells were used at the 12th passage.

\section{SAMPLE PREPARATION}

High RI (1.648) coverslips were coated with a $1.5 \mathrm{~nm}$ chro mium layer and a $45 \mathrm{~nm}$ gold layer by cathodic sputtering. $2.5 \times 10^{4}$ cells were seeded on the gold surface within their cul ture medium and kept $4 \mathrm{~h}$ at $37^{\circ} \mathrm{C}$ with $5 \% \mathrm{CO}_{2}$ to let them adhere on the gold substrate. The gold coated coverslip was then sealed to a second glass coverslip with a double face sticky spacer delimiting a rectangular chamber filled with the culture medium (Gene Frame from Thermo Fisher Scientific). To en able long term image acquisition with motionless biological ob jects, adherent myoblasts were fixed with $4 \%$ paraformaldehyde (PFA, Sigma) for $20 \mathrm{~min}$ at room temperature after rinsing the sample twice with PBS. Fixed myoblasts were covered with $25 \mu \mathrm{l}$ of phosphate buffered saline (PBS) while living myoblasts were maintained in $25 \mu \mathrm{l}$ of culture medium. The dimension and the volume of the chamber are $1 \mathrm{~cm} \times 1 \mathrm{~cm} \times 250 \mu \mathrm{m}$ and $25 \mu \mathrm{l}$. The time interval of $65 \mathrm{~s}$ between two images includes $10 \mathrm{~s}$ for $V(z)$ recording, $50 \mathrm{~s}$ for image acquisition, and a $5 \mathrm{~s}$ additional delay.

\section{$\boldsymbol{V}(\mathbf{z}) \mid$ COMPUTATIONS}

The theoretical $|V(Z)|$ curves were computed from the inte gral of the reflectivities $R_{p, s}(\theta)$ [Eq. (1)]. These reflectivities $R_{p}$ and $R_{s}$ were obtained from Fresnel equations for a 4 layer model comprising (i) a semi infinite coupling medium (1.57 NA Zeiss objective lens, High RI coverslip, and immersion oil) with RI $n_{1}=1.648$, (ii) a $45 \mathrm{~nm}$ gold layer with a com plex dielectric coefficient $\varepsilon_{2}(632.8 \mathrm{~nm})=12.31+1.22 i$, (iii) a dielectric layer with thickness $h$ and refractive index $n_{3}$, and (iv) a semi infinite liquid medium with refractive index $n_{4}=1.33$.

Funding. Agence Nationale de la Recherche (ANR) (IDEX 0007 02); AFM Telethon.

\section{REFERENCES}

1. A. L. Berrier and K. M. Yamada, "Cell matrix adhesion," J. Cell. Physiol. 213, 565573 (2007).
2. E. Zamir and B. Geiger, "Molecular complexity and dynamics of cell matrix adhesions," J. Cell Sci. 114, 35833590 (2001).

3. A. J. Ridley, "Life at the leading edge," Cell 145, 10121022 (2011).

4. R. O. Hynes, "The extracellular matrix: Not just pretty fibrils," Science 326, 12161219 (2009).

5. D. Choquet, D. P. Felsenfeld, and M. P. Sheetz, "Extracellular matrix rigidity causes strengthening of integrin cytoskeleton linkages," Cell 88, 3948 (1997).

6. C. K. Miranti and J. S. Brugge, "Sensing the environment: A historical perspective on integrin signal transduction," Nat. Cell Biol. 4, E83 E90 (2002).

7. S. W. Moore, P. Roca Cusachs, and M. P. Sheetz, "Stretchy proteins on stretchy substrates: The important elements of integrin mediated rigidity sensing," Dev. Cell 19, 194206 (2010).

8. A. Wagoner Johnson and B. A. C. Harley, Mechanobiology of Cell Cell and Cell Matrix Interactions (Springer Science, 2011).

9. R. Narayan, Biomedical Materials (Springer Science, 2009).

10. S. Burattini, P. Ferri, M. Battistelli, R. Curci, F. Luchetti, and E. Falcieri, " $\mathrm{C} 2 \mathrm{C} 12$ murine myoblasts as a model of skeletal muscle develop ment: Morpho functional characterization," Eur. J. Histochem. 48, 223233 (2004).

11. E. Ambrose, "A surface contact microscope for the study of cell move ments," Nature 178, 1194 (1956).

12. D. Axelrod, "Cell substrate contacts illuminated by total internal reflec tion fluorescence," J. Cell Biol. 89, 141145 (1981).

13. A. S. G. Curtis, "The mechanism of adhesion of cells to glass," A study by interference reflection microscopy," J. Cell Biol. 20, 199215 (1964).

14. H. Verschueren, "Interference reflection microscopy in cell biology methodology and applications," J. Cell Sci. 75, 279301 (1985).

15. M. R. Holt, Y. Calle, D. H. Sutton, D. R. Critchley, G. E. Jones, and G. A. Dunn, "Quantifying cell matrix adhesion dynamics in living cells using interference reflection microscopy," J. Microsc. 232, 7381 (2008).

16. J. Homola, S. S. Yee, and G. Gauglitz, "Surface plasmon resonance sensors: Review," Sens. Actuators B. 54, 315 (1999).

17. B. Rothenhausler and W. Knoll, "Surface plasmon microscopy," Nature 332, 615617 (1988).

18. K. F. Giebel, C. Bechinger, S. Herminghaus, M. Riedel, P. Leiderer, U. Weiland, and M. Bastmeyer, "Imaging of cell/substrate contacts of liv ing cells with surface plasmon resonance microscopy," Biophys. J. 76, 509516 (1999)

19. A. W. Peterson, M. Halter, A. Tona, K. Bhadriraju, and A. L. Plant, "Surface plasmon resonance imaging of cells and surface associated fibronectin," BMC Cell Biol. 10, 16 (2009).

20. A. W. Peterson, M. Halter, A. Tona, K. Bhadriraju, and A. L. Plant, "Using surface plasmon resonance imaging to probe dynamic inter actions between cells and extracellular matrix," Cytometry Part A 77A, 895903 (2010).

21. H. Kano, S. Mizuguchi, and S. Kawata, "Excitation of surface plasmon polaritons by a focused laser beam," J. Opt. Soc. Am. A 15, 1381 1386 (1998).

22. H. Kano and W. Knoll, "Locally excited surface plasmon polaritons for thickness measurement of LBK films," Opt. Commun. 153, 235239 (1998).

23. H. Kano and W. Knoll, "A scanning microscope employing localized surface plasmon polaritons as a sensing probe," Opt. Commun. 182, 1115 (2000).

24. M. G. Somekh, S. G. Liu, T. S. Velinov, and C. W. See, "Optical V(z) for high resolution 2 pi surface plasmon microscopy," Opt. Lett. 25, 823825 (2000).

25. M. G. Somekh, S. Liu, T. S. Velinov, and C. W. See, "High resolution scanning surface plasmon microscopy," Appl. Opt. 39, 62796287 (2000).

26. B. Zhang, S. Pechprasarn, J. Zhang, and M. G. Somekh, "Confocal surface plasmon microscopy with pupil function engineering," Opt. Express 20, 73887397 (2012).

27. B. Zhang, S. Pechprasarn, and M. G. Somekh, "Surface plasmon mi croscopic sensing with beam profile modulation," Opt. Express 20, 2803928048 (2012). 
28. B. Zhang, S. Pechprasarn, and M. G. Somekh, "Quantitative plas monic measurements using embedded phase stepping confocal inter ferometry," Opt. Express 21, 1152311535 (2013).

29. G. Stabler, M. G. Somekh, and C. W. See, "High resolution wide field surface plasmon microscopy," J. Microsc. (Oxford) 214, 328333 (2004).

30. B. Huang, F. Yu, and R. N. Zare, "Surface plasmon resonance imag ing using a high numerical aperture microscope objective," Anal. Chem. 79, 29792983 (2007).

31. T. Tanaka and S. Yamamoto, "Laser scanning surface plasmon polar iton resonance microscopy with multiple photodetectors," Appl. Opt. 42, 40024007 (2003).

32. L. Berguiga, S. Zhang, F. Argoul, and J. Elezgaray, "High resolution surface plasmon imaging in air and in water: V(z) curve and operating conditions," Opt. Lett. 32, 509511 (2007).

33. K. Watanabe, N. Horiguchi, and H. Kano, "Optimized measurement probe of the localized surface plasmon microscope by using radially polarized illumination," Appl. Opt. 46, 49854990 (2007).

34. T. Roland, L. Berguiga, J. Elezgaray, and F. Argoul, "Scanning sur face plasmon imaging of nanoparticles," Phys. Rev. B 81, 235419 (2010).

35. K. Watanabe, M. Ryosuke, G. Terakado, T. Okazaki, K. Morigaki, and $\mathrm{H}$. Kano, "High resolution imaging of patterned model biological membranes by localized surface plasmon microscopy," Appl. Opt. 49, 887891 (2010)

36. K. Watanabe, R. Miyazaki, G. Terakado, T. Okazaki, K. Morigaki, and H. Kano, "Localized surface plasmon microscopy of submicron do main structures of mixed lipid bilayers," Biomed. Opt. Express 3, 20122020 (2012).

37. M. M. A. Jamil, M. Youseffi, P. C. Twigg, S. T. Britland, S. Liu, C. W. See, J. Zhang, M. G. Somekh, and M. C. T. Denyer, "High resolution imaging of bio molecular binding studies using a widefield surface plasmon microscope," Sens. Actuators B 129, 566574 (2008).

38. M. G. Somekh, G. Stabler, S. Liu, J. Zhang, and C. W. See, "Wide field high resolution surface plasmon interference microscopy," Opt. Lett. 34, 31103112 (2009).

39. F. Sefat, M. C. T. Denyer, and M. Youseffi, "Imaging via widefield surface plasmon resonance microscope for studying bone cell inter actions with micropatterned ecm proteins," J. Microsc. 241, 282290 (2011).

40. L. Berguiga, T. Roland, K. Monier, J. Elezgaray, and F. Argoul, "Amplitude and phase images of cellular structures with a scanning surface plasmon microscope," Opt. Express 19, 65716586 (2011).

41. K. Watanabe, K. Matsuura, F. Kawata, K. Nagata, J. Ning, and H. Kano, "Scanning and non scanning surface plasmon microscopy to observe cell adhesion sites," Biomed. Opt. Express 3, 354359 (2012)

42. E. Boyer Provera, A. Rossi, L. Oriol, C. Dumontet, A. Plesa, L. Berguiga, J. Elezgaray, A. Arneodo, and F. Argoul, "Wavelet based decomposition of high resolution surface plasmon microscopy $V(Z)$ curves at visible and near infrared wavelengths," Opt. Express 21, 74567477 (2013).

43. K. J. Moh, X. C. Yuan, J. Bu, S. W. Zhu, and B. Z. Gao, "Surface plas mon resonance imaging of cell substrate contacts with radially polar ized beams," Opt. Express 16, 2073420741 (2008).

44. C. F. Soon, S. A. Khaghani, M. Youseffi, N. Nayan, H. Saim, S. Britland, N. Blagden, and M. C. T. Denyer, "Interfacial study of cell adhesion to liquid crystals using widefield surface plasmon resonance microscopy," Colloids Surf. B 110, 156162 (2013).
45. A. W. Peterson, M. Halter, A. Tona, and A. L. Plant, "High resolution surface plasmon resonance imaging for single cells," BMC Cell Biol. 15, 35 (2014).

46. M. M. A. Jamil, M. C. T. Denyer, M. Youseffi, S. T. Britland, S. Liu, C. W. See, M. G. Somekh, and J. Zhang, "Imaging of the cell surface interface using objective coupled widefield surface plasmon micros copy," J. Struct. Biol. 164, 7580 (2008).

47. W. Wang, K. Foley, X. Shan, S. Wang, S. Eaton, V. J. Nagaraj, P. Wiktor, U. Patel, and N. Tao, "Single cells and intracellular processes studied by a plasmonic based electrochemical impedance micros copy," Nat. Chem. 3, 249255 (2011).

48. W. Wang, S. Wang, Q. Liu, J. Wu, and N. Tao, "Mapping single cell substrate interactions by surface plasmon resonance microscopy," Langmuir 28, 1337313379 (2012).

49. K. Toma, H. Kano, and A. Offenhaeusser, "Label free measurement of cell electrode cleft gap distance with high spatial resolution surface plasmon microscopy," ACS Nano 8, 1261212619 (2014).

50. M. Stalder and M. Schadt, "Linearly polarized light with axial symmetry generated by liquid crystal polarization converters," Opt. Lett. 21, 19481950 (1996).

51. F. Argoul, T. Roland, A. Fahys, L. Berguiga, and J. Elezgaray, "Uncovering phase maps from surface plasmon resonance images: towards a sub wavelength resolution," Comptes Rendus Physique 13, 800814 (2012).

52. C. llett, M. G. Somekh, and G. A. D. Briggs, "Acoustic microscopy of elastic discontinuities," Proc. R. Soc. London Ser. A 393, 171183 (1984).

53. L. Berguiga, E. Boyer Provera, C. Martinez Torres, J. Elezgaray, A. Arneodo, and F. Argoul, "Guided wave microscopy: Mastering the in verse problem," Opt. Lett. 38, 42694272 (2013).

54. L. Berguiga, E. Boyer Provera, J. Elezgaray, and F. Argoul, "Sensing nanometer depth of focused optical fields with scanning surface plas mon microscopy," Plasmonics 8, 715722 (2013).

55. O. Loison and E. Fort, "Transmission surface plasmon resonance microscopy," Appl. Phys. Lett. 103, 133110 (2013).

56. T. Roland, A. Khalil, A. Tanenbaum, L. Berguiga, P. Delichere, L. Bonneviot, J. Elezgaray, A. Arneodo, and F. Argoul, "Revisiting the physical processes of vapodeposited thin gold films on chemically modified glass by atomic force and surface plasmon microscopies," Surf. Sci. 603, 33073320 (2009).

57. M. Morimatsu, A. H. Mekhdjian, A. C. Chang, S. J. Tan, and A. R. Dunn, "Visualizing the interior architecture of focal adhesions with high resolution traction maps," Nano Lett. 15, 22202228 (2015).

58. E. B. Magnusson, S. Halldorsson, R. M. T. Fleming, and K. Leosson, "Real time optical pH measurement in a standard microfluidic cell culture system," Biomed. Opt. Express 4, 17491758 (2013).

59. C. H. Choi, B. A. Webb, M. S. Chimenti, M. P. Jacobson, and D. L. Barber, "pH sensing by FAK His58 regulates focal adhesion remod eling," J. Cell Biol. 202, 849859 (2013).

60. D. Caballero, R. Voituriez, and D. Riveline, "Protrusion fluctuations direct cell motion," Biophys. J. 107, 3442 (2014).

61. S. Segawa, Y. Kozawa, and S. Sato, "Resolution enhancement of confocal microscopy by subtraction method with vector beams," Opt. Lett. 39, 31183121 (2014).

62. V. Yashunsky, V. Lirtsman, M. Golosovsky, D. Davidov, and B. Aroeti, "Real time monitoring of epithelial cell cell and cell substrate inter actions by infrared surface plasmon spectroscopy," Biophys. J. 99, 40284036 (2010).

63. V. Yashunsky, T. Marciano, V. Lirtsman, M. Golosovsky, D. Davidov, and B. Aroeti, "Real time sensing of cell morphology by infrared wave guide spectroscopy," PLoS One 7(10), e48454 (2012). 